\title{
The Fundamentals of Applying Electrospray Ionization Mass Spectrometry to Low Mass Poly(Methyl Methacrylate) Polymers
}

\author{
Charles N. McEwen, William J. Simonsick, Jr., and Barbara S. Larsen \\ E. I. du Pont de Nemours \& Company, P.O. Box 80228, Wilmington, Delaware, USA \\ Kaichi Ute and Koichi Hatada \\ Department of Chemistry, Faculty of Engineering Science, Osaka University, Toyonaka, Osaka, 560 Japan
}

\begin{abstract}
Electrospray ionization (ESI) is capable of ionizing many soluble polymers. The ESI spectra are complex because of overlap of the multiply charged ions of the oligomer distribution, causing current computer transform programs to fail. However, it is possible to determine the origin of the multiply charged ions, making it feasible to write a program designed to transform ESI polymer spectra. To assess the value of such a program for polymer analysis, isolated monodisperse methyl methacrylate (MMA) oligomers ( 25 and 50 repeat units) were used to determine molar signal response and propensity for fragmentation.

The sum of the peak areas for the multiply charged MMA 50-mer was found to be only about $66 \%$ of the summed peak areas for the 25 -mer for the same molar concentration. However, conversion of the multiply charged peak areas to the singly charged representations, with peak area compression taken into account, gave equal signal responses for the 25and 50-mers. Signal response variations due to the tacticity of the MMA oligomers were not observed. Fragmentation of the MMA oligomers also was shown not to occur under normal ESI conditions. Therefore, transformation of the polymer spectra to the singly charged molecular ion distribution should allow accurate calculation of average molecular weights, polydispersity, end group mass, and repeat unit mass. (I Am Soc Mass Spectrom 1995, 6. 906-911)
\end{abstract}

$\mathrm{M}$ ass spectrometry has been used extensively to analyze additives or impurities in polymers and to obtain ion patterns by pyrolysis or thermal degradation for polymer identification [1-3]. More recently, ionization methods have been developed that make characterization of intact polymers possible. These methods include field desorption (FD) $[4,5]$, liquid secondary-ion mass spectrometry (SIMS) (fast-atom bombardment) [6], SIMS [7, 8], laser desorption (LD) [9-11], potassium ionization of desorbed species (KIDS) $[12,13]$, electrospray ionization (ESI) [14], and matrix-assisted laser desorption ionization (MALDI) $[15,16]$. Even with the wide array of ionization methods potentially available for mass analysis of intact polymers, mass spectrometry seldom has been used as the definitive method to determine molecular weights and molecular weight distributions of polymers.

Address reprint requests to Charles N. McEwen, Central Research Department, E. I. du Pont de Nemours and Company, P.O. Box 80228, Wilmington, DE 19880-0228.
Mass spectrometers can provide absolute molecular weight. However, because polymers are mixtures of discrete compounds that differ in number and types of repeat units, end groups, architecture, and so forth, an accurate molecular weight analysis requires not only mass accuracy for each oligomer, but the absence of fragmentation and a signal response that is independent of oligomer mass or at least a known function of mass. Additionally, it would be advantageous to have no signal response differences associated with end groups, branching, stereochemistry, and so forth. Signal response discrimination can occur during any of the processes of ionization, transfer of ions to the mass analyzer, mass analysis, and ion detection.

Introduction of MALDI and ESI has considerably increased the mass range for molecular weight analyses by mass spectrometry. MALDI recently has been shown to be applicable to ionization of poly(styrenesulfonic acid)s that have molecular weight values around 400,000 [17]. Starburst dendrimers that have molecular weights above 1,000,000 have been detected with MALDI as doubly charged ions (Savickas, P., private communication). ESI has produced ions from 
polyethylene glycols (PEGs) that have molecular weights to 5,000,000 [14], although no useful information is available from electrospray data of high mass polymers because of the overlap of multiple charge states.

Dole et al. [18-20] were the first to attempt to apply ESI to polymers. Lnfortunately, experimental difficulties made Dole abandon the work and conclude that accurate molecular weights of polymers could not be determined by using electrospray ionization [21]. Fenn and co-workers [22] succeeded in interfacing ESI to a quadrupole mass spectrometer and they obtained mass spectra of PEGs [14]. Kalos et al. [23] used ESI to determine the molecular weight and polydispersity of polyamidoamine starburst dendrimers with a very narrow molecular weight distribution. In their work, a fully functionalized molecule had a molecular weight of 10632 , but, because of incomplete svnthesis, a narrow distribution with a polydispersity of 1.0007 of "oligomer" species that had +7 to +11 charges was observed. A composite molecular weight distribution was obtained by deconvolution of the distributions observed for each charge state. Prokai and Simonsick [24] produced a narrow distribution of octylphenoxypoly(ethoxy)ethanol oligomers [number average molecular weight $\left(M_{n}\right) 2000$ ] by coupling ESI with size-exclusion chromatography. These authors suggested that ions observed by ESI mass spectrometry for compounds that eluted from a gel permeation chromatography (GPC) column could be used as the low mass GPC calibrant.

ESI mass spectra of low molecular weight polymers have the potential to provide number-averaged molecular weight $\left(M_{n}\right)$, weight-averaged molecular weight $\left(M_{w}\right)$, polydispersity $(D)$, repeat unit mass, and end group mass:

$$
M_{n}=\frac{\sum M_{i} N_{i}}{\sum N_{i}} \quad M_{w}=\frac{\sum M_{i}^{2} N_{i}}{\sum M_{i} N_{i}} \quad D=\frac{M_{w}}{M_{n}}
$$

However, to obtain these values, it is necessary to transform the ESI mass spectrum of the multiply charged oligomer distribution to a distribution of singly charged oligomer molecular ions. The computer programs written to transform multiply charged protein molecular ions to the singly charged molecular ion can be applied to the mass spectra of low molecular weight polymers if each charge state oligomer distribution is resolved.

Before writing computer transform programs for polymer analysis by ESI, it is necessary to know that ESI provides a molar response to oligomers over a wide mass range and that fragmentation of oligomers does not occur under ESI conditions. Here we report the use of isolated monodisperse syndiotactic and isotactic $t$-butyl end-terminated poly(methyl methacrylate) (PMMA) oligomers (25- and 50-mers) to assess the propensity for oligomer fragmentation and to de- termine signal response as a function of oligomer mass and stereochemistry.

\section{Experimental}

The electrospray ion source (Analytica of Branford, Branford, CT) as previously described [25] was fitted to a ZAB-E double focusing high field mass spectrometer (Fisons Analytical, Manchester, UK). Data were collected on a Fisons 11-250 data system (PDP 11-73, Digital Equipment Corp., Maynard, CA) by using magnet scans at $30 \mathrm{~s} /$ decade with a resolution of 1000 $(10 \%$ valley). A minimum of 15 scans were summed in the multiple continuum acquisition (MCA) mode. Under these conditions, the total ion current for the methyl methacrylate (MMA) 25-mer relative to the 50-mers could be reproduced to better than $\pm 5 \%$. The data were then processed off-line on a VAX station 4000-60 (Digital Equipment) with OPUS (Fisons) software. Where stated, a Finnigan ISQ700 quadrupole mass spectrometer (Finnigan MAT, San Jose, CA) fitted with an Analytica of Branford electrospray source or a Fisons Trio 2000 quadrupole instrument fitted with a VG electrospray source (VG Analytical Ltd., Manchester, UK) were used to verify results. The sample liquid flow rate was maintained at $1 \mu \mathrm{L} / \mathrm{min}$ by using a Harvard syringe pump model 2000 (Harvard Apparatus, South Natick, MA).

The PMMA standards were acquired from Polymer Laboratories (Church Stretton, UK). The PEG standards were acquired from Aldrich Chemicals (Milwaukee, WI). The samples were run without further purification in methanol solutions at concentrations of $\sim 50 \mathrm{pmol} / \mu \mathrm{L}$. The individual MMA oligomers were isolated by supercritical fluid chromatography (SFC) as described previously [26, 27]. The isolated fractions were weighed, dissolved in chloroform, and diluted in 1:1 chloroform:methanol $\mathrm{v} / \mathrm{v}$ to equal concentrations of $\sim 20 \mathrm{pmol} / \mu \mathrm{L}$.

\section{Results and Discussion}

Figure la is an electrospray spectrum that shows multiply charged ions for PEG 3400 . Oligomers in the +2 charge state are reasonably well resolved, but peaks in the higher charge state distributions overlap. The transform algorithim requires a minimum of two charge states. The overlapping charge states are not recognized by the algorithim and no spectrum is produced. However, by increasing the voltage difference between the exit end of the capillary and the first skimmer, it is possible to change the appearance of the spectrum (Figure 1b) by charge stripping, which was first described by Musselman and co-workers [28]. When charge stripping conditions are used, the +1 , +2 , and +3 charge states are sufficiently resolved to transform the mass spectrum to the distribution of oligomers (Figure 1c). The transform programs cur- 


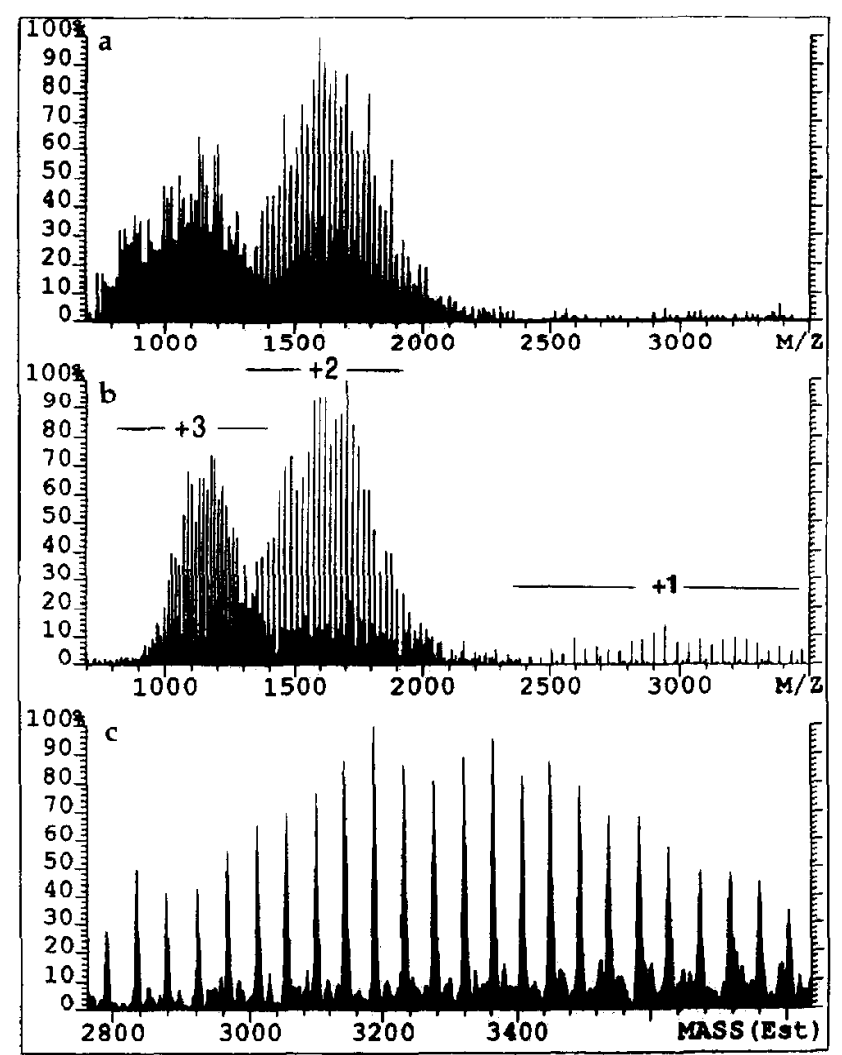

Figure 1. ESI mass spectra of PEG 3400. (a) Low capillary-toskimmer voltage. (b) Moderate capillary-to-skimmer voltage. (c) Transformation of spectrum (b)

rently available do not work well with the complex ESI spectra obtained for many polymers, especially when the spectrum contains multiple unresolved peaks.

Manual transformation of electrospray mass spectra of polymers to determine molecular weight distributions, repeat unit mass, and the mass of end groups is possible but requires an understanding of the origin of each multiply charged ion. Figure 2 is the ESI mass spectrum of a PMMA molecular weight standard with $\mathrm{Mp}=5270$, where $\mathrm{Mp}$ is the most probable mass, which is measured at the apex of the GPC polymer distribution curve. A series of peaks that have +1 through +4 charge states is observed (see Figure 2 inset). The repeat unit of PMMA is $100.1 \mathrm{u}$, and an ion series is observed with 100-u intervals between peaks (e.g., 1927, 2027, etc.). However, as will be shown subsequently, the large peaks that occur at 100-u intervals are actually a result of unresolved ions from different oligomer species that fall at nearly the same mass-to-charge ratio values due to multiple charging. This will occur for any homopolymer in which the end group mass is close to zero or a multiple of the mass of the repeat group. In this case, the PMMA is capped by hydrogen atoms.

Ionization in Figure 2 is by $\mathrm{Na}^{+}$addition to oligomers so that singly charged ions are observed at $100-u$ intervals [e.g., $(19 \times 100.1)+2+23=1927,(20$

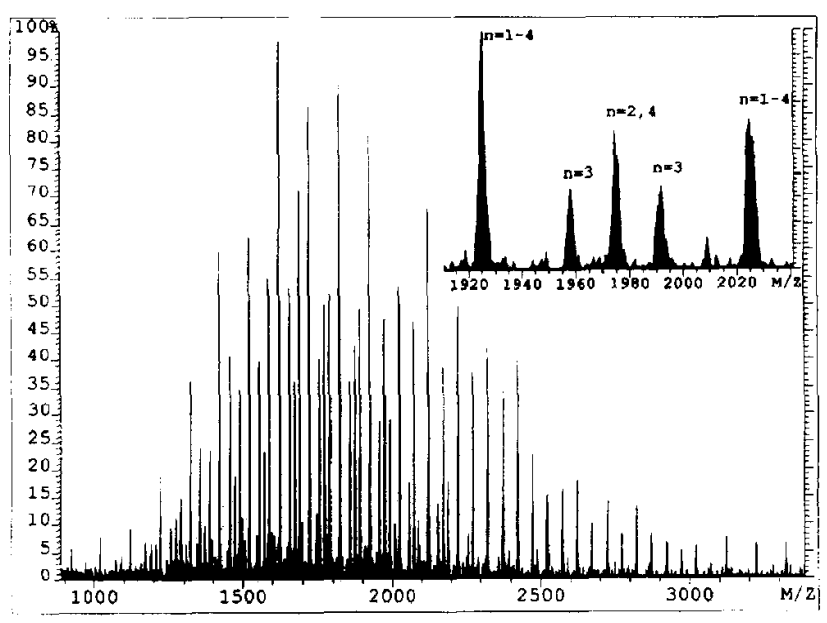

Figure 2. ESI mass spectrum of PMMA 5270. The inset is an expansion of mass range 1910-2040.

$\times 100.1)+2+23=2027$, etc.]. Ions of MMA oligomers with two charges $\left(2 \mathrm{Na}^{+}\right)$are observed every $50 \mathrm{~m} / z$ units. At the resolution employed, these will overlap ions that have one charge every $100 \mathrm{~m} / z$ units [e.g., $((38 \times 100.1)+2+(2 \times 23)) / 2=1926$, $((39 \times$ $100.1)+2+(2 \times 23)) / 2=1976,((40 \times 100.1)+2+$ $(2 \times 23)) / 2=2026)]$. Ions that have four charges $\left(4 \mathrm{Na}^{+}\right)$overlap ions that have two charges at $50 \mathrm{~m} / z$ unit intervals and singly charged ions at $100-\mathrm{u}$ intervals (e.g., 1975.5, 2000.5, 2025.5, etc.).

In the spectrum of Figure 2, the peaks observed at any mass-to-charge ratio are the sums of the ion current for the various multiply charged oligomers that fall in that mass-to-charge window. Because of the limited resolution of these experiments and because of peak broadening from ${ }^{13} \mathrm{C}$ isotopes, ions that fall within about a $2 \mathrm{~m} / \mathrm{z}$ window will be unresolved and contribute to the peak areas. Therefore, the peaks observed at $m / z 1926 \pm 1$ and $2026 \pm 1$ (Figure 2) are the sum of the peak areas from oligomers that have 1-4 charges, whereas the peak at $m / z 1976 \pm 1$ is derived from oligomers that have +2 and +4 charges. Use of this information and assumption of a smooth distribution of oligomers, make it possible to estimate the contribution of each charge state to a peak.

Determination of the molecular ion distribution for each of the charge states in the spectrum results in $M_{n}$ and $M_{w}$ values that are charge state dependent. The lowest charge state gives the lowest values and the highest charge state gives the highest $M_{\mathrm{n}}$ and $M_{\mathrm{w}}$ values. This is expected because the low $M_{n}$ oligomers can carry fewer charges than the higher $M_{\mathrm{n}}$ oligomers. Thus, it is not obvious which, if any, charge state gives the true molecular weight distribution or if a summation of the individual charge state distributions is accurate.

The absolute molecular weight that is obtained by mass spectrometry will provide good repeat unit and end group masses, but accurate values of $M_{n}$ and $M_{w}$ 
require that peak heights or peak areas be representative of the quantity of each oligomer present in the polymer. In addition, fragmentation of oligomer species that results in ions falling in the same mass-to-charge ratio range as the multiply charged oligomer distribution will complicate the spectra and could skew $M_{n}$ and $M_{w}$ to low values and result in misinterpretation of end groups. These issues are addressed by studying the ESI mass spectra of isolated monodisperse oligomers of PMMA.

\section{Molar Ion Response}

To assess the combined signal response versus oligomer mass (which reflects the efficiency of ionization, ion transmission, and ion detection), ESI mass spectra were obtained on equimolar solutions of isotactic (it) and syndiotactic (st) MMA 25-mers and 50-mers dissolved in chloroform and diluted with equal volumes of methanol.:<smiles>CCC(C)(CC)CC(C)(CC(C)(CC(C)C(=O)OC)C(=O)OC)C(=O)OC</smiles><smiles>COC(=O)C(C)CC(C)(CC(C)(CC(C)C)C(=O)OC)C(=O)OC</smiles>

The ion currents observed for the four isomers, run individually and in mixtures, allowed determination of molar signal response as a function of oligomer mass and tacticity. Results from the magnetic sector instrument are shown in Figure 3 for the st equimolar mixture of the 25-mer and 50-mer. Total combined ion current for the sum of all charge states of the 50-mer was $\sim 66 \%$ of the summed ion current for all charge states of the 25-mer obtained via peak area measurements. The same ratio was observed for an equimolar mixture of the it MMA 25-mer and 50-mer mix (Figure $4 a)$. Interestingly, summed peak areas for the it-50-mer were only $60 \%$ of the summed peak areas for the st-25-mer. The observed difference between 60 and $66 \%$ is outside of the experimental error for ESI reproducibility, but may be accounted for by the difficulties in weighing small samples after SFC purification.

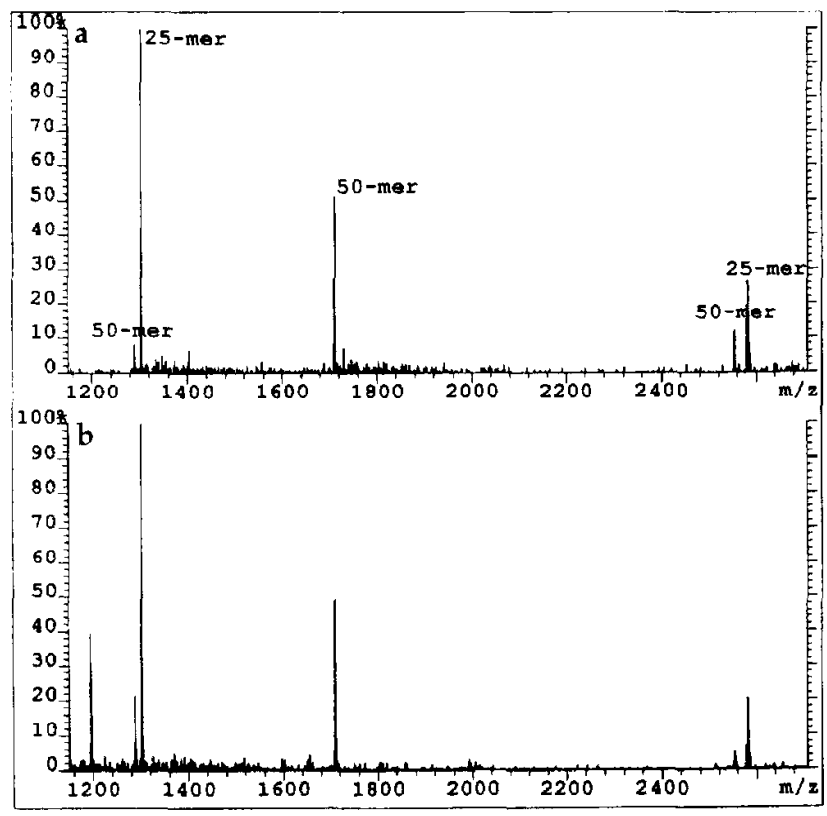

Figure 3. (a) ESI mass spectrum of an equimolar mixture of syndiotactic MMA 25-mer and 50-mer. (b) $5 \times$ dilution of the solution in spectrum (a).

These results demonstrate that for MMA oligomers that differ only in the number of repeat units, and thus mass, summation of the peak area of the multiply charged ions of each oligomer species discriminates against the higher mass oligomers. Approximately the same results were observed for quadrupole and magnetic sector instruments by using two different electrospray source designs. Thus, if the areas under the multiply charged peaks are used without compensation for charge state peak compression, the molecular weight distributions for polymers obtained by ESI will be skewed to low values.

Charge state peak compression occurs in ESI as a direct result of multiple charging. For example, a compound for which the isotope contribution causes a peak width at half-height of $6 \mathrm{~m} / z$ units when singly charged will have a half-width of $3 \mathrm{~m} / z$ units when doubly charged and only $2 \mathrm{~m} / \mathrm{z}$ units when triply charged. Thus, if it is assumed that the height of a peak in a mass spectrum corresponds to the number of ions in the peak regardless of the number of charges it carries, then the area under the peak is inversely proportional to the number of charges on the ions that comprise the peak. In other words, only one third the number of singly charged ions as triply charged ions are required to make peaks that have equal areas. Although this assumption may not be strictly true for actual data, it is a close approximation and is used in computer transform algorithms [29].

Application of the area corrections to the MMA oligomers gives approximately equal areas $( \pm 5 \%)$ for the equimolar st and it solutions shown in Figures 3 and 4 . Figure $3 \mathrm{~b}$ is a fivefold dilution of the solution used to obtain the spectrum in Figure 3a. The ion 


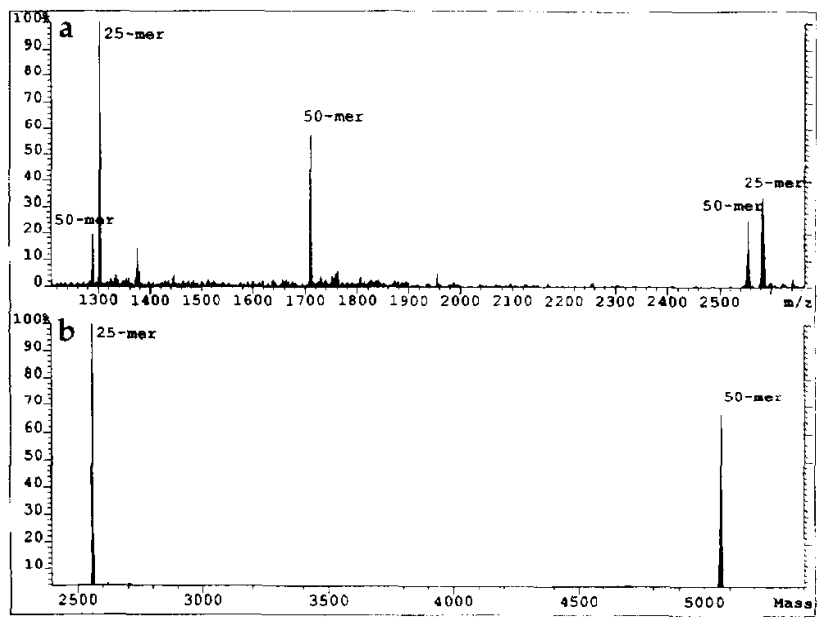

Figure 4. (a) ESI mass spectrum of an equimolar mixture of isotactic MMA 25-mer and 50-mer. (b) Transformation of the spectrum in (a).

current observed for the 25 -mer relative to the 50 -mer remained constant within the precision of the measurements. Figure $4 \mathrm{~b}$ shows the computer transform of the spectrum in Figure $4 a$. The peak height of the 50-mer is only $\sim 65 \%$ of the 25 -mer, but the areas under the transformed peaks are approximately equal.

This is the first direct evidence that ESI of oligomers can give a molar signal response on either magnetic or quadrupole mass spectrometers over a significant mass range. In the absence of fragmentation, ESI mass spectrometry will give reasonably accurate $M_{n}$ and $M_{w}$ for polymers in which the molar signal response discrepancy is small over a large mass range.

\section{Fragmentation}

Because the st and it oligomers contain no lower mass homologs, it was possible to assess propensity for fragmentation. As can be seen for the electrospray mass spectrum of the 25-mer (Figure 5a), the ion current for the molecular ion is dispersed into three charge states. The spectral appearance changes by raising the voltage difference between the capillary exit and the first skimmer on the vacuum side of the electrospray source. However, the only fragmentation observed for the individual oligomers of MMA (Figure 5b) was loss of $15 \mathrm{u}$ at the highest voltage $(350 \mathrm{~V})$ available for in-source fragmentation. An equivalent voltage difference was sufficient to cause extensive in-source fragmentation of peptides [30]. Fragmentation through an unzipping reaction that produces low mass fragment ions, which are then lost in transmission, cannot be ruled out. However, no fragmentation of the MMA oligomers was observed under standard tandem mass spectrometry operating conditions used for peptide fragmentation in a triple quadrupole mass spectrometer.

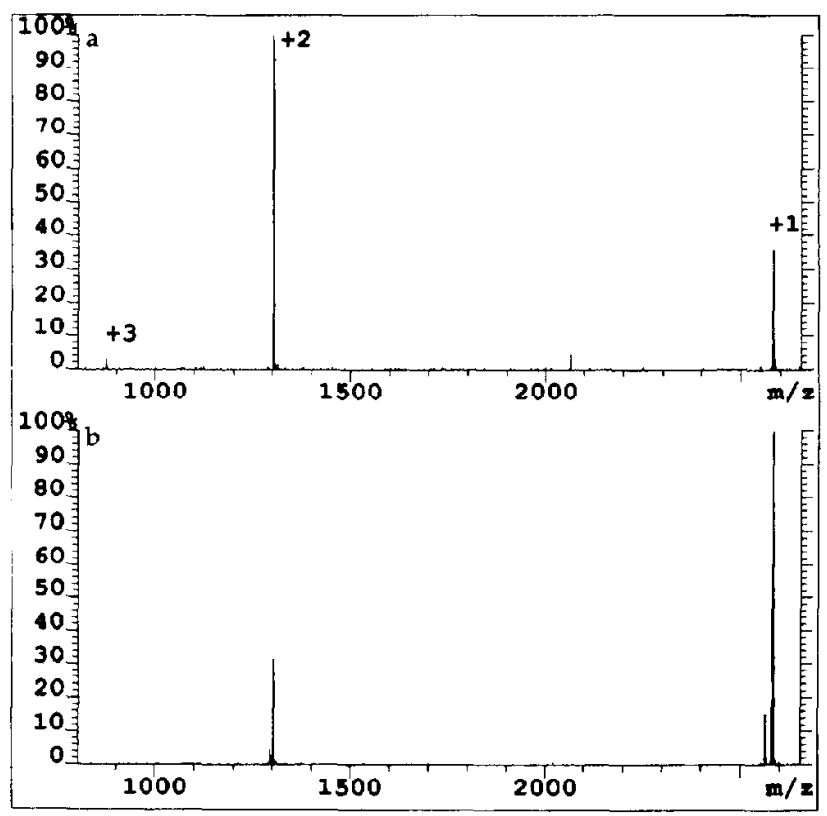

Figure 5. ESI mass spectra of isotactic MMA oligomer with 25 repeat units: (a) normal capillary-to-skimmer voltage; (b) high capillary-to-skimmer voltage.

These results show that for ions of MMA produced by ESI, fragmentation is difficult to achieve, at least in the mass range studied. The absence of fragmentation, in addition to simplifying the ESI mass spectrum, reduces the possibility of misinterpretation of end groups and removes fragmentation as a concern in determining $M_{n}$ and $M_{w}$ values.

\section{Stereochemistry}

Within the accuracy of these experiments, it is not possible via electrospray ionization to discern stereoisomers with respect to the total ion current observed from a given molar concentration. However, the number of charges that a given oligomer can accommodate might be effected by the stereochemistry. Proteins have shown this phenomenon in that folded proteins carry fewer charges than denatured and unfolded proteins [31]. For proteins, this is thought to be related to accessibility of basic sites for protonation.

With MMA oligomers, the cation $\left(\mathrm{Na}^{+}\right)$binds to oxygen atoms. The most stable configuration probably involves more than one oxygen atom in contact with a cation. Stereochemistry could disrupt the ability of the molecule to achieve the configuration needed for high charge states. This was not found to be the case because the analyte concentration and the voltage applied between the capillary exit and the skimmer were more important altering the abundances of charge states than effects of stereochemistry. It is possible, however, within limits, to determine the stereochemistry of MMA oligomers by using electron impact ionization mass spectrometry [32]. 


\section{Conclusion}

Although electrospray ionization of low mass polymers is complex because of the multiple charging of a distribution of oligomers, nevertheless, it can be deciphered to provide the oligomer distribution. Because fragmentation is demonstrated not to occur under normal electrospray conditions for MMA oligomers, it is possible to obtain repeat unit and end group masses. To obtain accurate molecular weight distributions by electrospray ionization it is required that the signal response be known for oligomers of different mass. For MMA oligomers that have 25 and 50 repeat units, it was demonstrated that equal signal response is obtained for equimolar concentrations of oligomers if the peak areas of the multiply charged peaks are properly transformed to the singly charged representation.

\section{References}

1. Vouros, P.; Wronka, J. W. In Modern Methods of Polymer Characterization; Barth, H.C.; Mays, J.W., Eds.; Wiley: Nen York, 1991; Chap. 12.

2. Cook, K. D. In Encyclopedia of Polymer Scionce and Engineering; 2nd ed.; Wiley: New York, 1987; Vol. 9. p 319.

3. Shulten, H-R.: Lattimer, R. P. Mass Spectrom. Reri. 1984, 3. 231.

4. Rollins, K.; Scrivens, I. H.; Taylor, M. J.; Major, H. Rapid Commun. Mass Spectrom. 1990, 4, 355.

5. Prokai, L. Field Desorption Mass Spettronetry; Marcel Dekker: New York, 1990; Vol 9

6. Cochran, R. L. Appl. Spectrosc. Riv. 1986, 22, 137.

7. van Leysen, D.; Hagenhoff. B.; Niehuis, E.; Benninghoven, A. Bletsos, I. V.; Hercules, D.M. I. Vac. Sci. Technol. A 1989, 1790 .

8. Hercules, D. Polym. Mat. Sa, 1988, 59, 724.

9. Tanaka, K.; Waki, H.; Ido, Y.; Akita, S.; Yoshida, Y.; Yoshida, T. Rapid Commun. Mass Sprectrom. 1988, 8, 151.

10. Nuwaysir, L. M.; Wilkins, C. L.; Simonsick, W. J., Jr. I. Am. Soc. Mass Spectrom. 1990, 1,66 71.

11. O'Malley, R. M.; Randazzo, M. E., Weinzicri, J. E.; Fernandez, J. E.; Nuwaysir, L. M.; Castora, I. A.; Wilkins, C. L. Maromolecules 1994, 27, 5107-5113.
12. Simonsick, W. J., Jr.; Hatada, K.; Xi, F.; Vogl, O. Macromolecules 1990, 23, 4470 .

13. Bombick, D.; Pinkston, J. D.; Allison, J., Anal. Chem. 1984, 56, 396.

14. Fenn, J. B.; Marn, M.; Meng, C. K.; Wong, S. F.; Whitehouse, C. M. Mass Spectrom. Rev. 1990, 9, 37.

15. Danis, P. O.; Karr, D. E.; Mayer, F.; Holle, A.; Watson, C. H. Org. Mass Spectrom. 1992, 27, 843.

16. Danis, P. O.; Karr, D. E. Org. Mass Spectrom. 1993, 28, 923.

17. Danis, P.O.; Karr, D. E.; Holle, A.; Mayer-Posner, F.; Watson, C. Proceedings of the 41st ASMS Conference on Mass Spectromeiry and Allied Topics; 1993, p 1093.

18. Dole, M.: Mack, L. L.; Hines, R. L.; Mobley, R. C.; Ferguson, L. D.; Alice, M. B. J. Chem. Phys. 1968, 49, 2240.

19. Dole, M.; Cox, H. L., Jr.; Giemic, J. J. Adv. Chem Ser. 1973, $125,73$.

20. Dole, M.; Gupta, C. V.; Mack, L. L.; Makamae, K. Polym. Prepr. An. Chem. Soc. Div. Polym. Chem. 1977, 18, 188.

21. Makamae, K.; Kumar, V.; Dole, M. Proceedings of the $29 t h$ ASMS Conference on Mass Spectrometry and Allied Topics; May 24-29, 1981, paper RPMOA3.

22. Wong, S. F.; Meng, C-K.; Fenn, J. B. I. Phys. Chem. 1988, 92 , 546.

23. Kallos, G. J.; Tomalia, D. A.; Hedstrand, D. M.; Lewis, S.; Zhou, J. Rapid Commun. Mass Spectrom. 1991, 5, 383.

24. Prokai, L.; Simonsick, W. J., Jr. Rapid Commun. Mass Spectrom. 1993, $7,853$.

25. Larsen, B. S.; McEwen, C. N. J. Am. Soc. Mass Spectrom. 1991, 2,205 .

26. Ute, K.; Miyatake, N.; Asada, T.; Hatada, K. Polym. Bull. 1992, 28, 561-568.

27. Ute, K.; Miyatake, N.; Osugi, Y.; Hatada, K. Polym. I. 1993, $25,1153 \cdots 1160$.

28. Cody, R. B.; Tamura, J; Musselman, B. D. Anal. Chem. 1992, $64,1561-1570$.

29. Mann, M.; Meng, C. K.; Fenn, J. B. Anal. Chen. 1989, 61, 1702-1708.

30. Meng, C. K.; McEwen, C. N.; Larsen, B. S. Rapid Commun. Mass Spectrom. 1990, 4, 151

31. Chowdhury, S. K.; Katta, V.; Chait, B. T. J. Am. Chem. Soc. $1990,112,9012$.

32. Kitayama, T.; Nishimura, T.; Oshima, A.; Ute, K.; Hatada, K. Polum. Bull. 1994, 32, 215-222. 